\title{
LncRNA FAM66C inhibits pancreatic cancer progression by sponging miR-574-3p
}

\author{
Jiangang Zhu, Sheng Zhu, Qiang Yu, Yong Wu \\ Department of Hepato-Biliary-Pancreatic Surgery, Nanjing Medical University Affiliated Changzhou No. 2 People’s Hospital, Changzhou 213000, \\ China \\ Contributions: (I) Conception and design: S Zhu; (II) Administrative support: None; (III) Provision of study materials or patients: None; (IV) \\ Collection and assembly of data: J Zhu, Q Yu; (V) Data analysis and interpretation: Y Wu; (VI) Manuscript writing: All authors; (VII) Final approval \\ of manuscript: All authors. \\ Correspondence to: Sheng Zhu. Department of Hepato-Biliary-Pancreatic Surgery, Nanjing Medical University Affiliated Changzhou No.2 People's \\ Hospital, Changzhou 213000, China. Email: zhusheng74@163.com.
}

\begin{abstract}
Background: Pancreatic cancer is an extensively concerned human malignancy around the globe, yet the potential therapeutic target remains to be further determined. MicroRNA and LncRNA have been reported to be involved in progression of pancreatic cancer, while the biological role of microRNA-574-3p (miR-574-3p) and FAM66C in pancreatic cancer development is poorly investigated.

Methods: Quantitative real-time PCR (qPCR) analysis was employed to detect the expression of miR574-3p and FAM66C in pancreatic normal or cancerous tissues and cells. The proliferative and apoptosis signaling molecules were also examined via qPCR and western blot separately. Additionally, cell proliferation and apoptosis assay were performed via CCK8, colony formation and Annexin V-FITC apoptosis assay. Interaction between miR-574-3p and FAM66C was interrogated by luciferase reporter assay and RNA immunoprecipitation. Even more, a pancreatic cancer xenograft mice assay was implemented to illustrate the coordinating role of miR-574-3p and FAM66C in pancreatic cancer proliferation.
\end{abstract}

Results: We found that levels of miR-574-3p were significantly higher in cancer tissues and cells compared to normal $(\mathrm{P}<0.05)$. Remarkably, the results indicated that depletion of miR-574-3p inhibited proliferation and promoted apoptosis of human pancreatic cancer cell lines. Additionally, FAM66C was demonstrated to interact with miR-574-3p and inhibit its expression. Significantly, FAM66C was proved to act as a tumor suppressor role via inhibiting cell proliferation and promoting cell apoptosis in pancreatic cancer. Moreover, FAM66C coordinated with miR-574-3p to regulate progression of xenograft tumor in the nude mice.

Conclusions: FAM66C-miR-574-3p axis mediates progression of pancreatic and might be the promising therapeutic target for pancreatic cancer patients.

Keywords: MiR-574-3p; FAM66C; Pancreatic cancer; LncRNA; Apoptosis.

Submitted Sep 22, 2019. Accepted for publication Jan 18, 2020.

doi: $10.21037 /$ tcr.2020.02.24

View this article at: http://dx.doi.org/10.21037/tcr.2020.02.24

\section{Introduction}

Pancreatic cancer is a highly aggressive cancer type, which ranks in the fourth leading cause for all human malignancies (1), and it is reported that patients with pancreatic cancer are frequently accompanied with poor survival rates owing to its metastasis and limited diagnosis and therapy methods (2-4). Correspondingly, despite that genetic alterations of several paramount molecules has been reported to be associated with progression of pancreatic cancer, for instance, K-Ras (5), INK4a (6), Tp53 (7) and SMAD4 (8), effective drugs targeting them are hardly realized for clinic due to the off-target effects or the drug resistance. Thus, demand to pursue novel diagnosis biomarkers or therapeutic targets for pancreatic cancer patients is pressing 
and molecular regulatory net on tumorigenesis of pancreatic cancer is expected to be further investigated.

MicroRNAs (miRNAs) characterized as an endogenous small molecule RNA (9) have been estimated to be involved in the progression of a series of cancers, including pancreatic cancer (10). Mechanistically, miRNAs negatively regulate post-transcriptional expression of genes through suppression of protein translation or mRNA degradation (11). It is reported that miRNA-96 suppresses KRAS inhibiting pancreatic cancer cell proliferation and invasion (12), and it is also shown that microRNA-1179 represses pancreatic cancer cell development via targeting E2F5 (13). Nevertheless, candidate regulatory miRNAs in pancreatic cancer is poorly explored.

In recent years, microRNA-574-3p (miR-574-3p) that is located in $4 \mathrm{p} 14$ has been found to be connected with development of several cancers (14). It has been reported that miR-574-3p acts as a tumor suppressor in bladder cancer (14), while it is also manifested that miR-574-3p could inhibit osteosarcoma progression by virtue of targeting SMAD4 (15). Accordingly, the detailed role of $\mathrm{miR}-574-3 \mathrm{p}$ in carcinogenesis needs to be further determined. Given that the molecular link between SMAD4 and pancreatic cancer is established, the role of $\mathrm{miR}-574-3 \mathrm{p}$ in the development of pancreatic cancer is expected to be illustrated. Emerging evidences have underscored that long non-coding RNAs (lncRNAs) with more than 200 nt act as multiple roles in human physiological and pathological conditions (16). Significantly, it is extensively known that lncRNAs regularly confer with miRNAs to function. It has been reported that $\operatorname{lncRNAs}$ behave as the sponge of miRNAs to negatively mediate their expression. For instance, PAGBC is proved to be the sponge of miR-133b and miR-511 microRNA sponge promoting gallbladder cancer development (17), and it is also shown that MAR1 plays as the sponge of miR-487b to be implicated in skeletal muscle differentiation and regeneration (18). Hence, understanding the lncRNAs-miRNAs regulatory axis is of great importance to the progression of diseases. However, to date, few $\operatorname{lncRNAs}$ on miR-574-3p are investigated.

In this time research, we revealed the detailed role of miR-574-3p in pancreatic cancer. Remarkably, we showed that miR-574-3p was upregulated in pancreatic cancer tissues and cells via promoting cancer cell proliferation and inhibiting apoptosis. Additionally, FAM66C was demonstrated to interact with miR-574-3p and acted in concert with it to mediate progression of pancreatic cancer, hinting that FAM66C-miR-574-3p axis might be a putative therapeutic target for patients with pancreatic cancer.

\section{Methods}

\section{Cell culture and transfection}

Human CFPAC-1 and HPAC cell lines were purchased from the American Type Culture Collection (ATCC, United States) and cultured in DMEM (HyClone, United States) medium supplemented with $10 \%$ fetal bovine serum (Gibco, United States) and 1\% penicillin/streptomycin (HyClone, United States). All cells were cultured in a humidified incubator equilibrated with $5 \% \mathrm{CO}_{2}$ at $37{ }^{\circ} \mathrm{C} ; \mathrm{MiR}-574-3 \mathrm{p}$ inhibitor, miR-574-3p mimics, siRNA of FAM66C and the negative control sequences were purchased from GenePharma (Shanghai, China). The sequences of FAM66C containing the putative binding sites of miR574-3p were synthetized from Sangon Biotech (Shanghai, China) and cloned into the pmirGLO Dual-Luciferase vector, and the FAM66C overexpression vector was also built into pcDNA3.1 vector via Sangon Biotech. All cells were transfected with Lipofectamine 3000 (Thermo, United States) according to the manufacturer's recommendation. Lentiviral production and infection were implemented by Sangon Biotech (Shanghai, China) on CFPAC-1 cells. Each experiment was performed in triplicate and repeated at least 3 times. The detailed sequences $\left(5^{\prime}-3^{\prime}\right)$ were shown in Table 1.

\section{$R N A$ extraction and quantitative real-time PCR (qPCR)}

TRIzol and chloroform were employed to purify RNA from tissues or cells. Briefly, tissues or cells were lysed with TRIzol and the supernatant was collected with isopropanol for further purification. RNA precipitates were obtained after centrifugation and then dissolved with nuclease-free water; Reverse transcription with RT-PCR kit (Transgene, China) was used to synthesize the cDNA according to the manufacturer's instruction; The relative mRNA expression was detected by LightCycler480 II Sequence Detection System (Roche, Switzerland) and GAPDH or U6 was employed as the internal normalizer. The results were calculated using $2^{-\Delta \Delta c t}$ method. The sequences of the primers used were shown in Table 2.

\section{Western blot analysis}

Tissues were ground and then lysed with RIPA lysis buffer supplemented with proteinase inhibitors cocktail 
Table 1 Mimic and inhibitor of miR-424-5p and siRNA sequences of FAM66C

\begin{tabular}{ll}
\hline Genes and RNAs & Sequence $\left(5^{\prime}-3^{\prime}\right)$ \\
\hline FAM66C & CACCUGCGGAGACUUUAAAUU \\
miR-574-3p-mimic & CACGCUCAUGCACACACCCACA \\
miR-574-3p-inhibitor & CACGCUCAUGCACACACCCACA \\
\hline
\end{tabular}

Table 2 Primers of qPCR

\begin{tabular}{ll}
\hline Genes and RNAs & Primers (5'-3') \\
\hline FAM66C & F: AGGAAGCCTGAGGTGTAGCA; R: CACTCTCCCACCCATCACTT \\
miR-574-3p & F: CACGCTCATGCACACA; R: GTGCAGGGTCCGAGGT \\
PCNA & F: CCTGCTGGGATATTAGCTCCA; R: CAGCGGTAGGTGTCGAAGC \\
CDK2 & F: CCAGGAGTTACTTCTATGCCTGA; R: TTCATCCAGGGGAGGTACAAC \\
Cyclin A1 & F: GAGGTCCCGATGCTTGTCAG; R: GTTAGCAGCCCTAGCACTGTC \\
Bax & F: CCCGAGAGGTCTTTTTCCGAG; R: CCAGCCCATGATGGTTCTGAT \\
TP53 & F: CAGCACATGACGGAGGTTGT; R: TCATCCAAATACTCCACACGC \\
Caspase-3 & F: CATGGAAGCGAATCAATGGACT; R: CTGTACCAGACCGAGATGTCA \\
U6 & F: CTCGCTTCGGCAGCACA; R: AACGCTTCACGAATTGCGT \\
GAPDH & F: TCGGAGTCAACGGATTTGGT; RTCCCGTTCTCAGCCTTGAC \\
\hline
\end{tabular}

(Roche, Switzerland) for $30 \mathrm{~min}$. These proteins were then separated by $10 \%$ SDS-PAGE. After that, proteins were transferred to NC membrane (Pall Corporation, United States) for $90 \mathrm{~min}$ with $400 \mathrm{~mA}$. Membranes were blocked with $5 \%$ skim milk for $1 \mathrm{~h}$ at room temperature and the indicated primary antibodies were used for incubation at $4{ }^{\circ} \mathrm{C}$ overnight, including PCNA, CDK2, Cyclin A1, TP53, Bax, Cleaved-Caspase- 3 and GAPDH, which were all from Proteintech. The next day, membranes with incubation of primary antibodies were washed with TBST and then incubated with secondary antibodies (Proteintech, China) for $1 \mathrm{~h}$ at room temperature. Luminol buffer from Santa were used for the exposure.

\section{Luciferase reporter assay}

The miR-574-3p binding sequences FAM66C and the mutant were constructed into pmirGLO Dual-Luciferase vector to build the FAM66C-wild type and FAM66Cmutant plasmids. And then plasmids were transfected into pancreatic cancer cell cells together with scramble, miR-574-3p mimics or anti-miR- miR-574-3p. The relative luciferase activity was calculated by the Dual-Glo Luciferase
Assay System according to manufacturer's suggestions (Promega, United States).

\section{Cell proliferation assay}

CCK8 (MedChem Express, China) was used to detect the cell proliferative capacity according to the manufacturer's guide. Briefly, pancreatic cancer cells were seeded into 96well plates and $10 \mu \mathrm{L}$ CCk- 8 solution was added at the indicated times. Then the cell viability was determined by measuring the absorbance at wavelengths $450 \mathrm{~nm}$ using a Microplate Reader (Bio Tek, Bio Tek).

\section{Colony formation assay}

Pancreatic cancer cells were plated in six-well plates and incubated for 2 weeks. Then the colonies were washed, fixed with $4 \%$ formaldehyde and stained with $0.1 \%$ crystal violet.

\section{Cell apoptosis assay}

The Annexin V-FITC Apoptosis Detection Kit (Abcam, BioTek) was used to test the apoptosis of pancreatic 
A

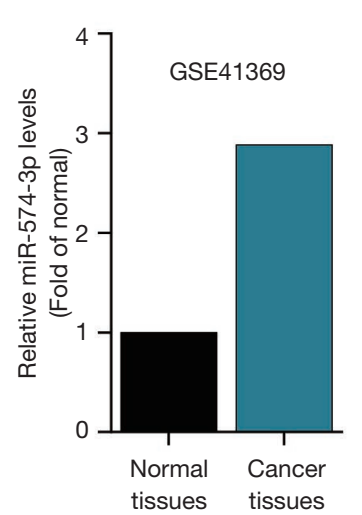

B

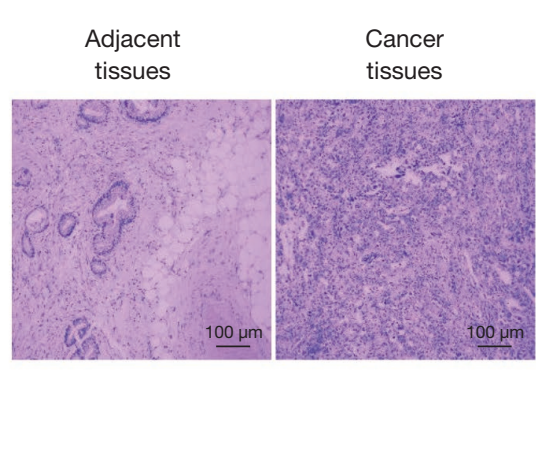

C

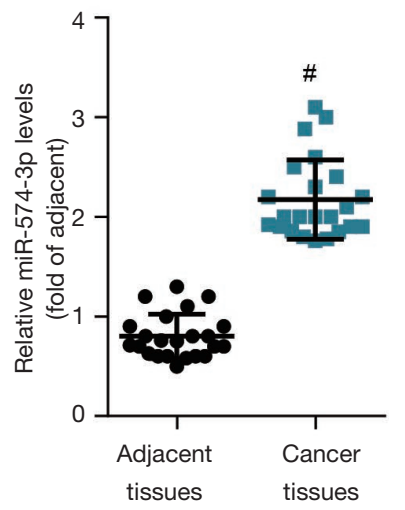

D



Figure 1 MiR-574-3p was upregulated in pancreatic cancer tissues and cells. (A) Interrogation of GSE41369 by GEO2R. (B) HE staining showed the morphological changes of pancreatic cancer tissues. (C) Expression of miR-574-3p in pancreatic cancer and adjacent tissues was detected by qPCR, ${ }^{\#} \mathrm{P}<0.01$ represents the comparison to adjacent tissues. (D) Expression of miR-574-3p in pancreatic cancer and normal cells was detected by qPCR, ${ }^{\#} \mathrm{P}<0.01$ represents the comparison to HPC-Y5 cells.

cancer cell based on the manufacturer's instructions with a flow cytometer (FACSCalibur, Becton-Dickinson, United States). Data were analyzed by Cell-Quest software.

\section{Xenograft}

Male C57BL/6 nude mice with 6-8-week were purchased from Charles River (Beijing, China). Animal study have been approved by animal Ethics Committee of Nanjing Medical University (Approval number: NMU-2018$365)$ and performed according to guidelines. CFPAC-1 $\left(2 \times 10^{6}\right.$ cells $)$ cells were implanted into the flanks of nude mice. After 4 weeks, tumors were resected and measured for volumes.

\section{Statistical analysis}

All data in this study were represented as the mean \pm standard deviation (mean \pm SD) and analyzed by SPSS 22.0 by a $t$-test. A P value less than 0.05 was considered to be statistically significant.

\section{Results}

\section{MiR-574-3p was upregulated in pancreatic cancer tissues and cells}

To address the role of miR-574-3p in the progression of pancreatic cancer, GEO2R analysis was employed to examine the expression of $\mathrm{miR}-574-3 \mathrm{p}$ in pancreatic cancer and normal tissues initially with the interrogation of GSE41369. The results showed that miR-474-3p were upregulated in cancer tissues (Figure 1A). We then determined the mRNA expression level of miR-574-3p between cancer and its adjacent tissues that was confirmed by $\mathrm{H} \& \mathrm{E}$ staining (Figure 1B). Our data found that miR-574-3p was significantly higher in cancer tissues than normal tissues (Figure 1C). Further interrogation of miR-574-3p expression across pancreatic cell lines revealed that it had a higher mRNA levels in pancreatic cancer cells than normal cells (Figure 1D). Taken together, these data hinted that miR-574-3p might exert role of contributing to pancreatic cancer progression.

\section{Depletion of miR-574-3p inbibited proliferation and promoted apoptosis of pancreatic cancer cells}

To further delineate the biological function of miR-574-3p in pancreatic cancer progression, the role of miR-574-3p in pancreatic cancer cell proliferation and apoptosis was investigated. Given the high expression of miR-574-3p in pancreatic cancer, inhibitor of miR-574-3p was used to examine the effects of it on cellular behaviors. Scramble RNA and anti-miR-574-3p were transfected into pancreatic cancer CFPAC-1 and HPAC cell lines and the knockdown efficiency was detected by qPCR. As the data shown (Figure 2A,B), the anti-miR-574-3p functioned remarkably 
in these two cell lines, cell proliferation and apoptosis analysis were then implemented in both CCK8 and colony formation experiments were employed to examine the cell proliferative capacity. With the knockdown of miR-574-3p, cell viability of CFPAC- 1 and HPAC cells decreased contrast to control group (Figure $2 C, D$ ). Similarly, colony numbers were reduced overtly after transfection of antimiR-574-3p (Figure 2E,F). Since PCNA (19), CDK2 (20) and Cyclin A1 (21) have been reported to be proliferative markers that represented the cell proliferative capacity, mRNA levels of them were detected when the miR-574-3p was depleted. The data showed that mRNA levels of these proteins declined significantly after transfection of anti-miR-574-3p both in CFPAC-1 and HPAC cell lines (Figure 2G,H).

To further clarify the effects of miR-574-3p on pancreatic cancer development, apoptosis assay on CFPAC-1 and HPAC cells was performed. After cells were transfected with anti-miR-574-3p for $48 \mathrm{~h}$, they were collected and analyzed by flow cytometry. We found that apoptosis rates were only about $1 \%$ in control group, whereas the apoptosis rates increased to nearly $8 \%$ after knockdown of miR-574-3p (Figure 2I,f). Consistently, mRNA levels of apoptosis markers including Bax, TP53 and Caspase-3 elevated with the transfection of anti-574-3p (Figure $2 K, L$ ). Taken together, our results indicated that miR-574-3p might contribute to pancreatic cancer progression via promoting cell proliferation and inhibiting cell apoptosis.

\section{FAM66C regulated expression of miR-574-3p}

To further explore the molecular mechanisms of miR-574-3p in progression of pancreatic cancer, its potential interactors were screened. Previous researches have found that that miRNAs interacted with lcnRNAs and functionally interwined. Hence, it would be a safe assumption that one putative lncRNA interacted with miR-574-3p and is functionally connected with miR-574-3p to be involved in pancreatic cancer progression. By virtue of online bioinformatics tool, we found a significant negative correlation between lncRNA FAM66C and miR-574-3p $(\mathrm{r}=-0.331, \mathrm{P}=6.46 \mathrm{e}-06)$ (Figure $3 A$ ).

To further demonstrate the association between FAM66C and miR-574-3p, we constructed the wild-type (WT) and mutant (Mut) FAM66C luciferase reporter plasmids that contained the wild-type and mutant binding sites of miR-574-3p, respectively (Figure 3B). Correspondingly, the WT and Mut luciferase reporter plasmids were transfected together with control RNA, miR-574-3p mimics and antimiR-574-3p to the CFPAC-1 and HPAC cells, and the luciferase activity was measured. The results showed that the luciferase activity decreased after overexpression of miR-574-3p and elevated with the transfection of antimiR-574-3p in WT group, whereas the difference was completely counteracted when the mutant plasmids were transfected into cells (Figure 3C).

To further corroborate the association between FAM66C and miR-574-3p, RNA immunoprecipitation (RIP) assay with the antibodies against IgG and Argonaute2 (Ago2) was implemented in CFPAC-1 and HPAC cells. The results showed that levels of FAM66C and miR-574-3p in Ago-2 immunoprecipitates were significantly higher than IgG group in both of cells, which further sustained the functional link between miR-574-3p and FAM66C (Figure 3D,E). Moreover, we found that levels of miR-574-3p decreased and increased after overexpression and knockdown of FAM66C separately (Figure 3F), suggesting that FAM66C might negatively regulate expression of $\mathrm{miR}-574-3 \mathrm{p}$ as a competing endogenous RNA (ceRNA). Additionally, with the transfection of miR-574-3p mimics and anti-574-3p into the CFPAC-1 and HPAC cells, levels of FAM66C also decreased and increased, respectively (Figure 3G), hinting a potential reciprocal regulation between FAM66C and miR-574-3p. Taken together, our data suggested that FAM66C could sponge the miR-574-3p and negatively mediate its expression at the molecular level.

\section{FAM66C inbibited proliferation and promoted apoptosis of pancreatic cancer cells}

As stated above, FAM66C was identified as the ceRNA of miR-574-3p and miR-574-3p was verified to be a tumor promoter in pancreatic cancer cells, then it was a plausible assumption that FAM66C could suppress the progression of pancreatic cancer. Initially, online tool GEPIA was used to address the survival time of pancreatic patients with differential expression of FAM66C. The data showed that the lower FAM66C expression was significantly associated with a poorer overall survival rates in pancreatic patients $(\mathrm{P}=0.037, \mathrm{HR}=0.65)$ (Figure 4A). Correspondingly, we found that levels of FAM66C were significantly lower in pancreatic cancer tissues compared to its adjacent tissues (Figure 4B), which further supported the tumor suppressor role in pancreatic cancer.

To further clarify the function of FAM66C in pancreatic cancer, the effects of FAM66C on pancreatic 
A

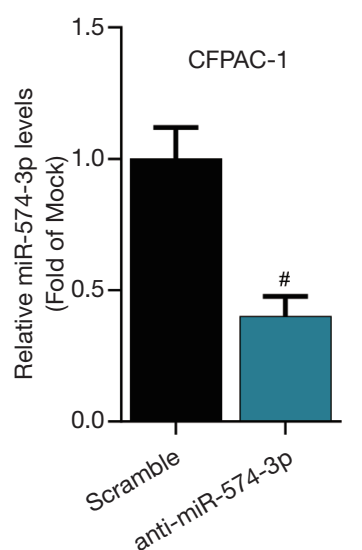

$E$



I



$\bar{\alpha}$
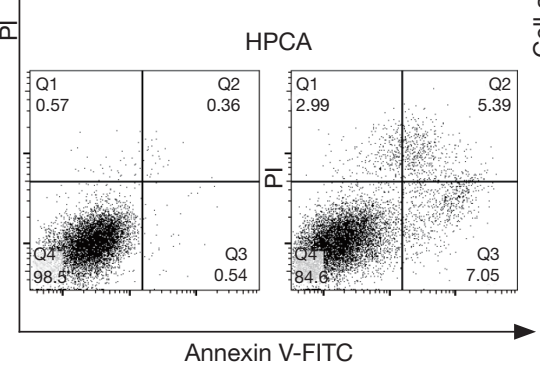

C

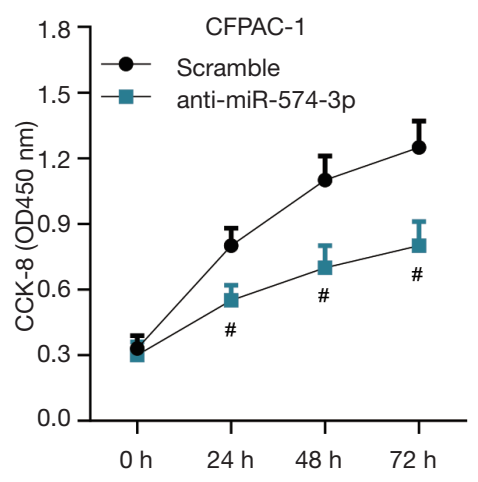

$\mathrm{D}$

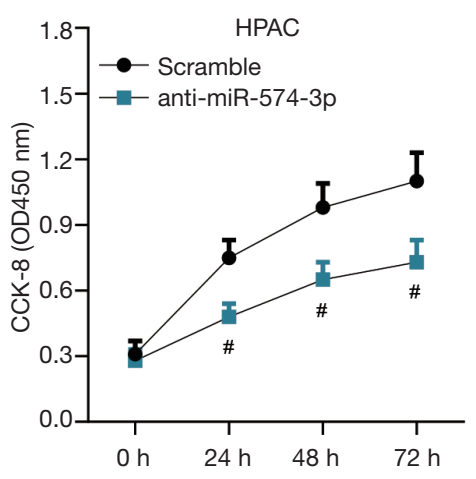

Figure 2 Depletion of miR-574-3p inhibited proliferation and promoted apoptosis of pancreatic cancer cells. (A,B) qPCR was used to detect the expression of miR-574-3p after the transfection of anti-miR-574-3p; (C,D) CCK8 assay was used to detect the cancer cell viability after the transfection of anti-miR-574-3p; (E,F) Colony formation assay was used to detect the cancer cell proliferative capacity after the transfection of anti-miR-574-3p; (G,H) Expression of proliferative related genes was detected by qPCR after the transfection of anti-miR574-3p; (I,J) Annexin V-FITC/PI staining assay was used to examine the cancer cell apoptosis after the transfection of anti-miR-574-3p; (K,L) Expression of apoptosis related genes was detected by qPCR after the transfection of anti-miR-574-3p. ${ }^{\#} \mathrm{P}<0.01$ represents the comparison to scramble group. 
A

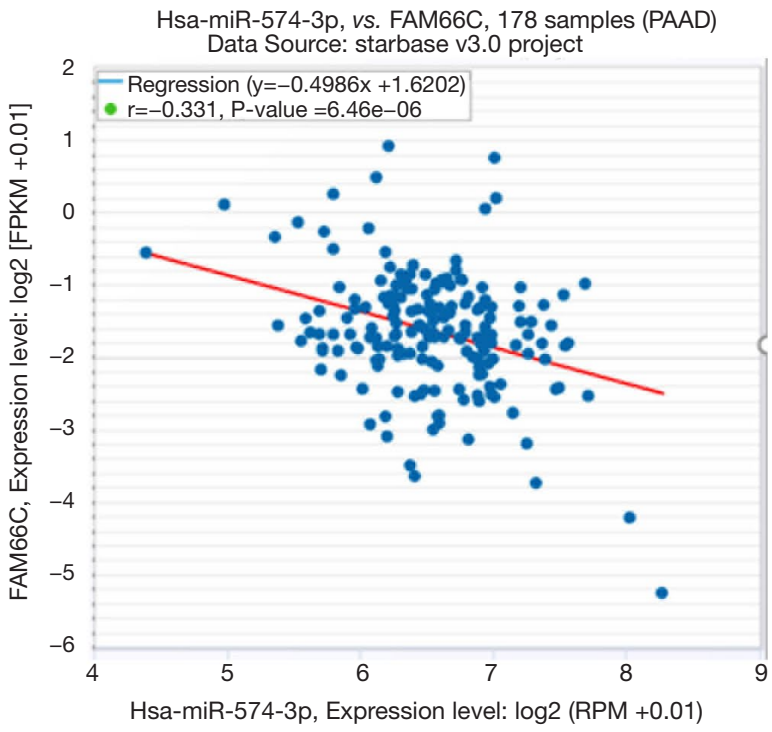

C

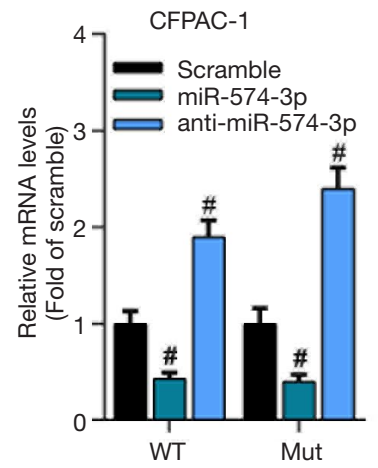

E





WB: Ago2
B

Binding sites of miR-574-3p on

FAM66C: Predication by DIANA tools

FAM66C Mut 5'-CUUUCACGCU-3'

AGUAACAGAAUAAAUUUA

GUAUGAGCGU

|. $|1||||| \mid$

CGUACUCGCA

$$
\text { A }
$$

$\mathrm{F}$

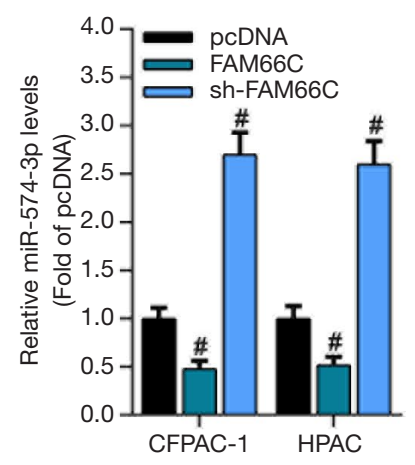

G

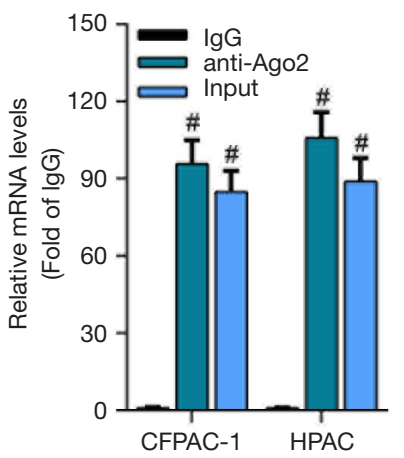

Figure 3 FAM66C regulated expression of miR-574-3p. (A) Correlation between the expression of FAM66C and miR-574-3p was analyzed with the online bioinformatics tool (STARBASE). (B) Binding site of miR-574-3p on FAM66C was shown. (C) Luciferase reporter assay was used to detect luciferase activity of FAM66C with overexpression and knockdown of miR-574-3p, ${ }^{\#} \mathrm{P}<0.01$ represents the comparison to control group. (D,E) RIP assay was examined by qPCR, ${ }^{*} \mathrm{P}<0.01$ represents the comparison to IgG group. (F) Expression of miR-574-3p was examined by qPCR after overexpression and knockdown of FAM66C, ${ }^{~} \mathrm{P}<0.01$ represents the comparison to control group. (G) Expression of FAM66C was examined by qPCR after overexpression and knockdown of miR-574-3p, ${ }^{\#} \mathrm{P}<0.01$ represents the comparison to control group.

cancer cell proliferation and apoptosis were investigated. Considering the low expression of FAM66Cin pancreatic cancer, plasmids containing the wild-type FAM66C were transiently transfected into cells to examine the effects on cellular behaviors. With the overexpression of FAM66C, we uncovered that cell viability was reduced overtly via CCK8 assay in CFPAC-1 and HPAC cells (Figure 4C,D).
In addition, colony formation was also employed to detect proliferative capacity of pancreatic cancer cells and the results revealed that colony numbers decreased after the transfection of FAM66C (Figure 4E,F). Moreover, mRNA levels of PCNA, CDK2 and Cyclin A1 was measured by qPCR and the data showed that their expression declined notably with the gain function of FAM66C in both of 
A

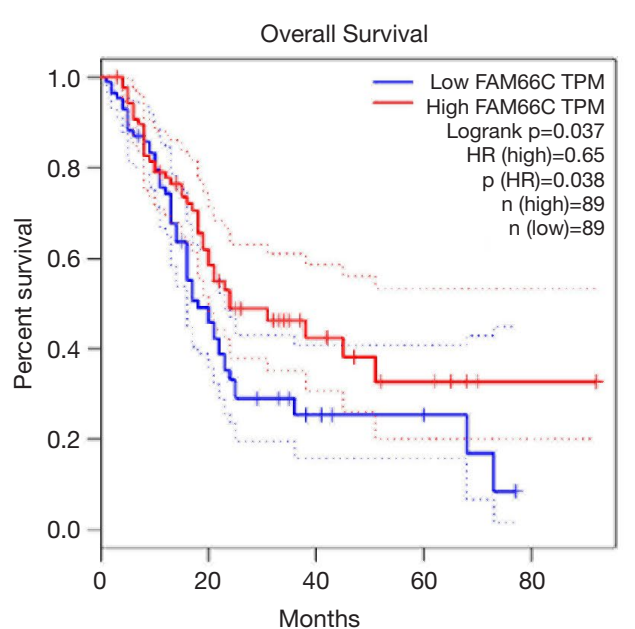

B

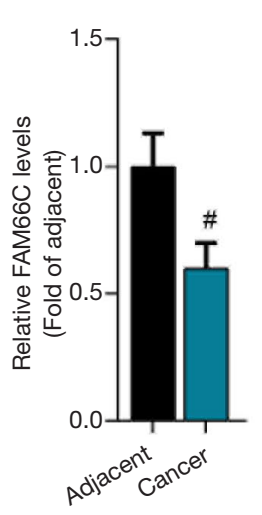

C

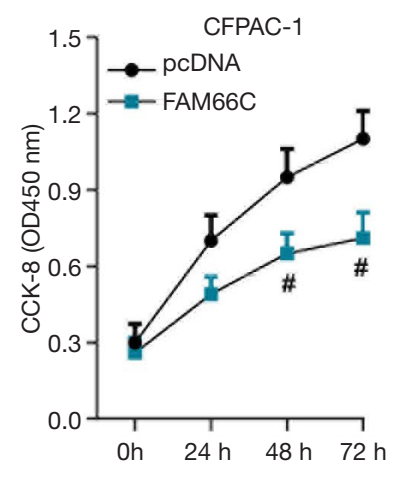

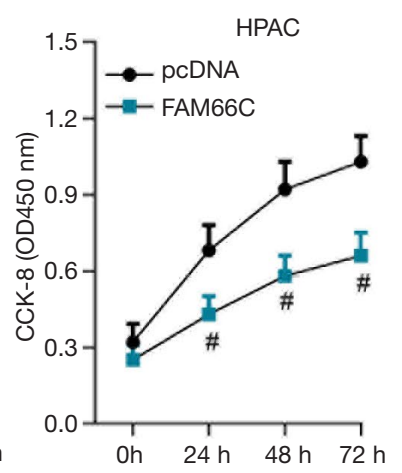

$\mathrm{E}$
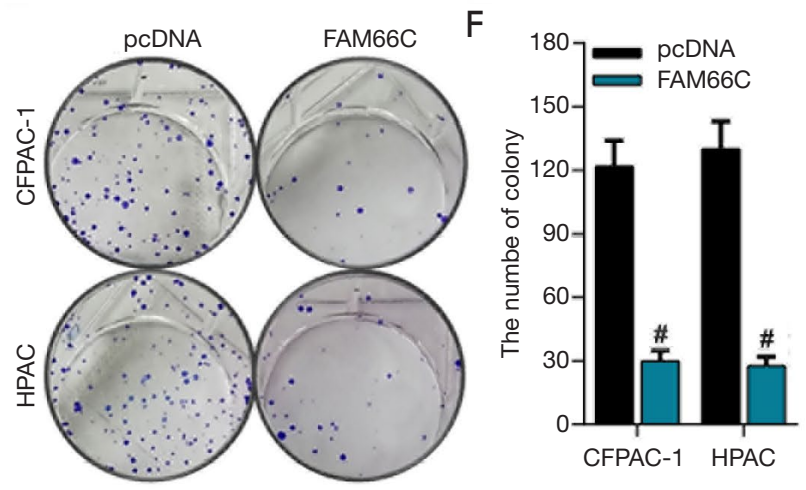

G
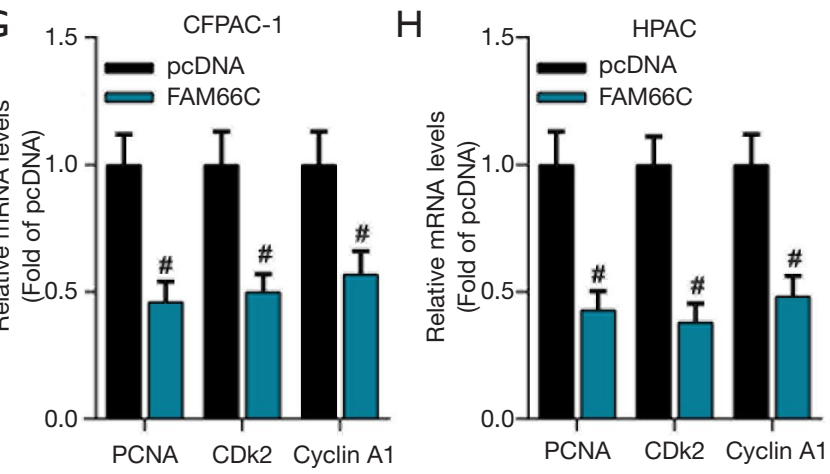

I



K

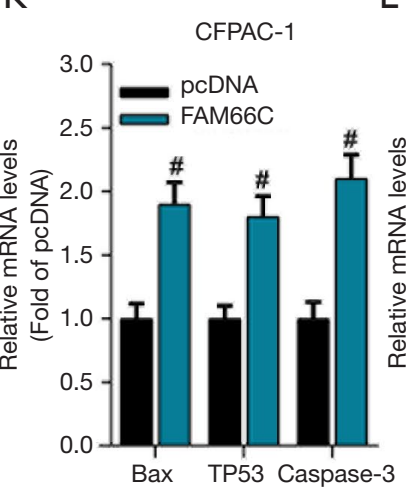

L

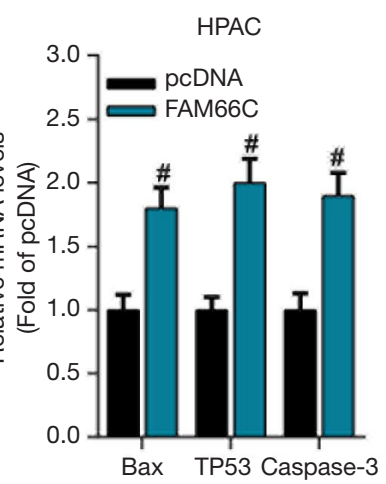

Figure 4 FAM66C inhibited proliferation and promoted apoptosis of pancreatic cancer cells. (A) Correlation between FAM66C and prognosis of cancer patients was analyzed by GEPIA tool. (B) Expression of FAM66C in pancreatic cancer and adjacent tissues was detected by qPCR. (C,D) CCK8 assay was used to detect the cancer cell viability after the transfection of FAM66C. (E,F) Colony formation assay was used to detect the cancer cell proliferative capacity after the transfection of FAM66C. $(\mathrm{G}, \mathrm{H})$ Expression of proliferative related genes was detected by qPCR after the transfection of FAM66C. (I,J) Annexin V-FITC/PI staining assay was used to examine the cancer cell apoptosis after the transfection of FAM66C. (K,L) Expression of apoptosis related genes was detected by qPCR after the transfection of FAM66C. ${ }^{\#} \mathrm{P}<0.01$ represents the comparison to control group. 
cells (Figure 4G,H). Next, cell apoptosis was detected and we found that apoptosis rates elevated over 6-fold to control group in CFPAC-1 and HPAC cells (Figure 4I,7). Additionally, we also examined the expression of Bax, TP53 and Caspase- 3 and the data revealed that their mRNA levels increased remarkably through the overexpression of FAM66C in both of cells (Figure 4K,L). Taken together, our data sustained that FAM66C might be a bona fide tumor suppressor via inhibiting cell proliferation and promoting cell apoptosis of pancreatic cancer.

\section{FAM66C coordinated with miR-574-3p to orchestrate the tumor growth of xenograft in nude mice}

As mentioned above, FAM66C and miR-574-3p were functionally intertwined at the molecular level and both were involved in the progression of pancreatic cancer, we then reasoned that they might coordinate with each other in the development of pancreatic cancer. To confirm this hypothesis, nude mice xenograft assays were performed in CFPAC-1 cells that were transfected with lentiviruses carrying vector and scramble RNA or lenti-miR-574-3p and lenti-FAM66C together with lenti-miR-574-3p. Four weeks later, the tumors were dissected and their volume and weight was measured, respectively. The results showed that tumor volume and weight decreased significantly after the overexpression of miR-574-3p (Figure $5 A, B, C$ ), which was consistent with the role of miR-574-3p in pancreatic cancer cells. Importantly, further overexpression of FAM66C in CFPAC-1 cells could partially neutralize the inhibitory effects (Figure $5 A, B, C$ ), which further sustaining the role of FAM66C in suppressing the pancreatic cancer development.

Additionally, we detected the mRNA and protein levels of proliferative and apoptosis markers separately in the tumor tissues. The data showed that mRNA and protein levels of PCNA, CDK2 and Cyclin A1 was reduced via the overexpression of miR-574-3p, which could be offset by further overexpression of FAM66C (Figure 5D,E). Oppositely, mRNA and protein levels of Bax, TP53 and Caspase-3 elevated overtly after transfection of miR-574$3 \mathrm{p}$, which could be rescued after together transfection of FAM66C (Figure 5D,E). Taken together, our data suggested that FAM66C could work in concert with miR-574-3p to regulate the tumor growth of xenograft in nude mice.

\section{Discussion}

Emerging evidence has revealed that miRNAs exert crucial roles in the tumorigenesis of several cancers. It is reported that miR-362 could inhibit cell proliferative and invasive capacity of cervical cancer (22), and it is also shown that miR19b-3p acts as a tumor suppressor in colorectal cancer via targeting ITGB8 (23). Across these miRNAs, miR-574$3 p$ has been extensively accepted as a tumor suppressor role in cancers, for instance, breast cancer (24), gastric cancer (25) and bladder cancer (14), while it also has been documented to be a tumor promoter in osteosarcoma (15). Clearly, the role of miR-574-3p in cancer remains to be further explored. Notably, rare study is investigated on miR574-3p in pancreatic cancer and its underlying molecular mechanism is largely elusive.

In this time study, we revealed a tumor promoter role of miR-574-3p in pancreatic cancer for the first time, which was consistent to the previous study (15). Our data found that miR-574-3p was upregulated in pancreatic cancer tissues and cells, and it contributed to cancer progression probably via accelerating cell proliferation and inhibiting cell apoptosis. Significantly, these results not only hint a putative role of miR-574-3p as the diagnostic or prognostic role of pancreatic cancer patients in clinic, but also support the potential therapeutic target for the clinical patients.

Massive studies have showed that miRNAs and lncRNAs are functionally intertwined and it is broadly known that lncRNAs act as the sponge of the miRNAs to inhibit their function. For instance, it is reported that lncRNA H19 promotes the colorectal cancer cell invasive capacity by sponging miRNAs (26). Thus, to further delineate the molecular mechanism of miR-574-3p in pancreatic cancer, its potential sponge lncRNA was figured out by virtue of bioinformatics tool. Intriguingly, our results revealed a novel lncRNA, FAM66C, which was significantly correlated with miR-574-3p with poor research. Importantly, FAM66C was verified to be the sponge of miR-574-3p and negatively mediate its expression. Notably, we found that a reciprocal regulation might exist between FAM66C and miR-574-3p cause that levels of FAM66C increased after depletion of miR-574-3p. Additionally, we also uncovered the biological role of FAM66C in the development of pancreatic cancer and our data showed that FAM66C inhibited proliferation and promote apoptosis of pancreatic cancer cells. Moreover, with the xenograft tumor assay in nude mice, coordination between FAM66C and miR-574-3p was observed in the progression of pancreatic cancer. These results also provide a novel biomarker for patients with pancreatic cancer. Momentously, the authenticated FAM66C-miR-574-3p signaling axis confers more therapeutic choice for clinical 
A

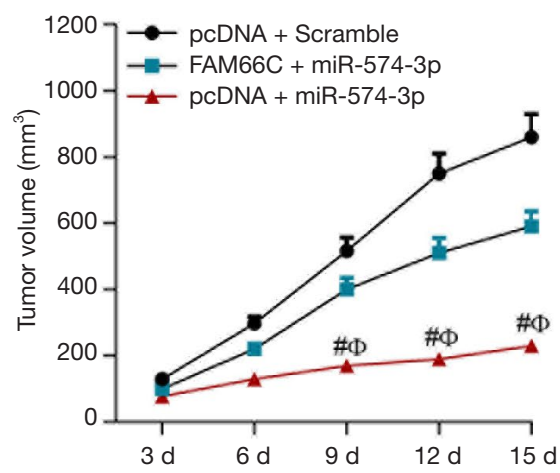

B

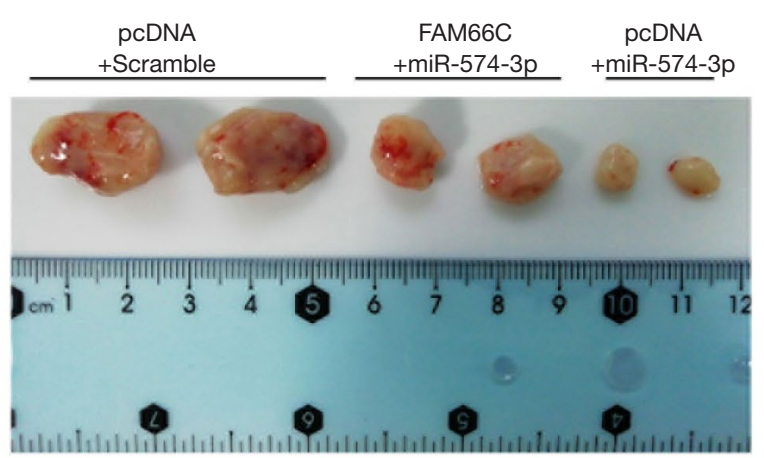

C

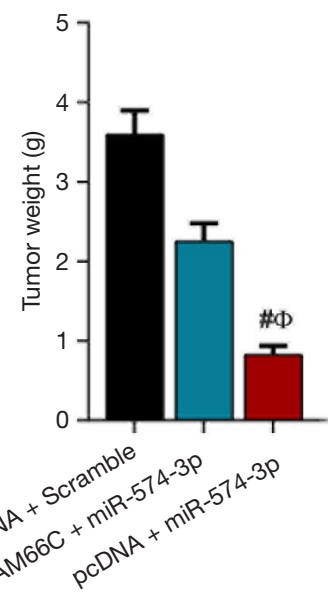

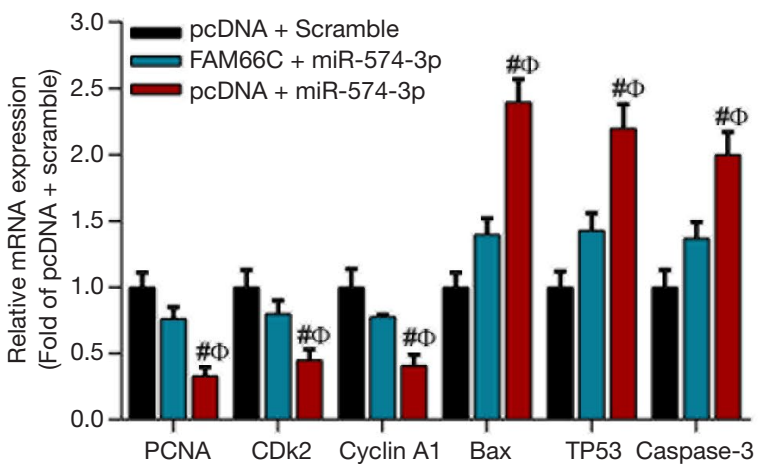

E

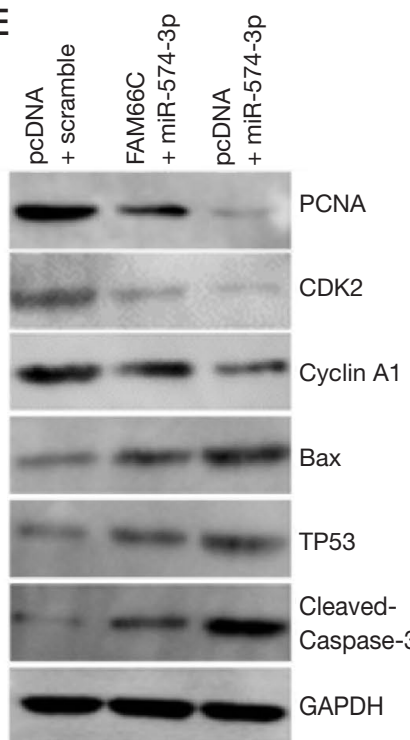

Figure 5 FAM66C coordinated with miR-574-3p to orchestrate the tumor growth of xenograft in nude mice. (A) Tumor volumes was measured after 4 weeks; (B) tumor was shown; (C) tumor weight was measured after 4 weeks; (D) proliferative and apoptosis related genes expression was examined by qPCR in tumor tissues; (E) proliferative and apoptosis related protein expression was examined by qPCR in tumor tissues. ${ }^{~} \mathrm{P}<0.01$ represents the comparison to pcDNA + scramble group; ${ }^{\Phi} \mathrm{P}<0.01$ represents the comparison to FAM66C + miR-574-3p group.

pancreatic cancer patients.

Nevertheless, considering the multiple cellular function of FAM66C and miR-574-3p, their role in pancreatic cancer remains to be further explored in the future study. Moreover, the potential target of miR-574-3p in the pancreatic cancer is expected to be further investigated.

In conclusion, we report here that IncRNA FAM66C inhibits pancreatic cancer progression by sponging miR-574$3 p$, revealing a novel FAM66C-miR-574-3p signaling axis in the progression of pancreatic cancer and also the putative diagnostic markers and therapeutic targets for patients.

\section{Acknowledgments}

Funding: None.

\section{Footnote}

Conflicts of Interest: All authors have completed the ICMJE uniform disclosure form (available at http://dx.doi. 
org/10.21037/tcr.2020.02.24). The authors have no conflicts of interest to declare.

Ethical Statement: The authors are accountable for all aspects of the work in ensuring that questions related to the accuracy or integrity of any part of the work are appropriately investigated and resolved. The study was conducted in accordance with the Declaration of Helsinki (as revised in 2013). Informed consent was waived. Animal study have been approved by animal Ethics Committee of Nanjing Medical University (Approval number: NMU2018-365) and performed according to guidelines for the care and use of animals.

Open Access Statement: This is an Open Access article distributed in accordance with the Creative Commons Attribution-NonCommercial-NoDerivs 4.0 International License (CC BY-NC-ND 4.0), which permits the noncommercial replication and distribution of the article with the strict proviso that no changes or edits are made and the original work is properly cited (including links to both the formal publication through the relevant DOI and the license). See: https://creativecommons.org/licenses/by-nc-nd/4.0/.

\section{References}

1. Hariharan D, Saied A, and Kocher HM. Analysis of mortality rates for pancreatic cancer across the world. HPB (Oxford) 2008;10:58-62.

2. Liu $\mathrm{Y}, \mathrm{Wu} \mathrm{T}, \mathrm{Lu} \mathrm{D}$, et al. CD44 overexpression related to lymph node metastasis and poor prognosis of pancreatic cancer. Int J Biol Markers 2018;33:308-13.

3. Mo Z, Hu M, Yu F, et al. Leukemia-related protein 16 (LRP16) promotes tumor growth and metastasis in pancreatic cancer. Onco Targets Ther 2018;11:1215-22.

4. Paulson AS, Tran Cao HS, Tempero MA, et al. Therapeutic advances in pancreatic cancer. Gastroenterology 2013;144:1316-26.

5. Hamada S, Taguchi K, Masamune A, et al. Nrf2 promotes mutant K-ras/p53-driven pancreatic carcinogenesis. Carcinogenesis 2017;38:661-70.

6. Zhang H, Li X, Meng W, et al. Overexpression of p16(ink4a) regulates the $\mathrm{Wnt} /$ beta-catenin signaling pathway in pancreatic cancer cells. Mol Med Rep 2018;17:2614-8.

7. Mello SS, Valente LJ, Raj N, et al. A p53 Super-tumor Suppressor Reveals a Tumor Suppressive p53-Ptpn14Yap Axis in Pancreatic Cancer. Cancer Cell 2017;32:460- 473.e6.

8. Ormanns S, Haas M, Remold A, et al. The Impact of SMAD4 Loss on Outcome in Patients with Advanced Pancreatic Cancer Treated with Systemic Chemotherapy. Int J Mol Sci 2017;18:1094-104.

9. Shukla GC, Singh J, Barik S. MicroRNAs: Processing, Maturation, Target Recognition and Regulatory Functions. Mol Cell Pharmacol 2011;3:83-92.

10. Xiong G, Huang H, Feng M, et al. MiR-10a-5p targets TFAP2C to promote gemcitabine resistance in pancreatic ductal adenocarcinoma. J Exp Clin Cancer Res 2018;37:76-90.

11. Bartel DP. MicroRNAs: target recognition and regulatory functions. Cell 2009;136:215-33.

12. Sivakumar S, de Santiago I, Chlon L, et al. Master Regulators of Oncogenic KRAS Response in Pancreatic Cancer: An Integrative Network Biology Analysis. PLoS Med 2017;14:e1002223.

13. Lin C, Hu Z, Yuan G, et al. MicroRNA-1179 inhibits the proliferation, migration and invasion of human pancreatic cancer cells by targeting E2F5. Chem Biol Interact 2018;291:65-71.

14. Tatarano S, Chiyomaru T, Kawakami K, et al. Novel oncogenic function of mesoderm development candidate 1 and its regulation by MiR-574-3p in bladder cancer cell lines. Int J Oncol 2012;40:951-9.

15. Xu H, Liu X, Zhou J, et al. miR-574-3p acts as a tumor promoter in osteosarcoma by targeting SMAD4 signaling pathway. Oncol Lett 2016;12:5247-53.

16. Mercer TR, Dinger ME, and Mattick JS. Long noncoding RNAs: insights into functions. Nat Rev Genet 2009;10:155-9.

17. Wu XS, Wang F, Li HF, et al. LncRNA-PAGBC acts as a microRNA sponge and promotes gallbladder tumorigenesis. EMBO Rep 2017;18:1837-53.

18. Zhang ZK, Li J, Guan D, et al. A newly identified lncRNA MAR1 acts as a miR-487b sponge to promote skeletal muscle differentiation and regeneration. J Cachexia Sarcopenia Muscle 2018;9:613-26.

19. Hall PA, Levison DA, Woods AL, et al. Proliferating cell nuclear antigen (PCNA) immunolocalization in paraffin sections: an index of cell proliferation with evidence of deregulated expression in some neoplasms. J Pathol 1990;162:285-94.

20. Gopinathan L, Tan SL, Padmakumar VC, et al. Loss of Cdk2 and cyclin A2 impairs cell proliferation and tumorigenesis. Cancer Res 2014;74:3870-9.

21. Ji P, Agrawal S, Diederichs S, et al. Cyclin A1, 
the alternative A-type cyclin, contributes to G1/ $\mathrm{S}$ cell cycle progression in somatic cells. Oncogene 2005;24:2739-44.

22. Shi C, and Zhang Z. MicroRNA-362 is downregulated in cervical cancer and inhibits cell proliferation, migration and invasion by directly targeting SIX1. Oncol Rep 2017;37:501-9.

23. Huang L, Cai JL, Huang PZ, et al. miR19b-3p promotes the growth and metastasis of colorectal cancer via directly targeting ITGB8. Am J Cancer Res 2017;7:1996-2008.

Cite this article as: Zhu J, Zhu S, Yu Q, Wu Y. LncRNA FAM66C inhibits pancreatic cancer progression by sponging miR-574-3p. Transl Cancer Res 2020;9(3):1806-1817. doi: 10.21037/tcr.2020.02.24
24. Krishnan P, Ghosh S, Wang B, et al. Next generation sequencing profiling identifies miR-574-3p and miR-660$5 \mathrm{p}$ as potential novel prognostic markers for breast cancer. BMC genomics 2015;16:735-51.

25. Zhang R, Wang M, Sui P, et al. Upregulation of microRNA-574-3p in a human gastric cancer cell line AGS by TGF- $\beta 1$. Gene 2017;605:63-9.

26. Liang WC, Fu WM, Wong CW, et al. The LncRNA H19 promotes epithelial to mesenchymal transition by functioning as MiRNA sponges in colorectal cancer. Oncotarget 2015;6:22513-25. 